LUIZ ROGÉRIO WITTMANN

UNIVERSIDADE DE SÃO PAULO, USP

SÃO PAULO, BRAZIL

ROGERIOWITTMANN@USP.BR

DENISE DANTAS

UNIVERSIDADE DE SÃO PAULO, USP,

SÃO PAULO, BRAZIL.

DEDANTAS@USP.BR
Fecha de recepción: 29/06/2020

Fecha de aceptación: 21/09/2020

Cómo citar: Wittmann, R. \& Dantas, D. (2020)

Diseño de embalajes y pandemia: Cambios

en el sector alimentación en Brasil.

RChD: creación y pensamiento, 5(9), 1-13.

https://doi.org/10.5354/0719-837X.2020.57795

\section{Diseño de envases y la pandemia: Cambios en el sector alimentación en Brasil}

\author{
Packaging Design and Pandemic: Changes in the Food \\ Sector in Brazil
}

Resumen. Este artículo realiza un análisis de noticias e investigaciones relacionadas con los cambios de hábitos de consumo de productos alimenticios en Brasil durante el período que se extiende de marzo a julio de 2020, cuando el mundo enfrentó la pandemia de Covid-19. Se buscan identificar los cambios en el comportamiento de los consumidores en el sector alimenticio con el fin de proponer nuevas soluciones de Diseño. Para ello, se realizó una investigación bibliográfica exploratoria en sitios de noticias, órganos gubernamentales, institutos de investigación, revistas especializadas y webinars, seguida de un análisis descriptivo de los resultados obtenidos. Como conclusión, se presenta un cuadro que describe la situación actual en Brasil relacionado con los cambios identificados en los hábitos de consumo y las posibles soluciones que pueden ser empleadas en nuevos diseños de envases de alimentos que satisfacen las necesidades de los consumidores en una situación de pandemia, aislamiento social y restricciones de movilidad.

Palabras clave: Diseño de embalaje, embalaje, hábitos de consumo, pandemia Covid-19

Abstract. This paper analyzies news and research related to changes in the consumption habits of food products in Brazil from March to July 2020, during which the world has been facing the Covid-19 pandemic. It seeks to identify changes in consumer behavior in the food sector in order to propose new design solutions. To achieve that, exploratory bibliographic research was carried out on news sites, government agencies, research institutes, journals and webinars, followed by a descriptive analysis of the results. As a conclusion, a table presents the current situation in Brazil, listing the changes identified in consumption habits and the possible solutions that may be used for new food packaging designs that satisfy the needs of consumers in situations of pandemic, social isolation, and mobility restrictions.

Keywords: Consumer behavior, Covid-19 pandemic, packaging design. 


\section{Introducción}

La pandemia causada por Covid-19 alcanzó a 9.129.146 de personas en todo el mundo, $y$ ha ocasionado la muerte de 473.797 personas hasta el día 24 de julio de 2020 (Organización Mundial de la Salud, 2020). Brasil, el país más afectado de América Latina y el tercero en casos confirmados, totaliza 1.145.906 casos hasta el 23 de junio de 2020 (Ministerio de Salud, 2020). Este hecho provocó que los gobiernos estatales decretasen medidas de aislamiento social para contener el avance del virus. La cuarentena impuesta obligó a la sociedad a adaptarse a este escenario, inédito para todas las generaciones. De esta situación crítica emergieron nuevos hábitos y comportamientos que influenciaron al sector de los envases. Solo las actividades consideradas como esenciales podían funcionar, entre ellas, los supermercados, farmacias, gasolineras, el sector de la construcción civil y la industria de alimentos y productos esenciales. Se cerraron oficinas y centros comerciales, las clases se suspendieron, mientras que los bares y los restaurantes solo podían atender a sus clientes por medio del servicio de delivery. Esta situación generó un nuevo escenario, sin precedentes en Brasil. Por un lado, sectores como el automotriz tuvieron que interrumpir su producción. Por otro lado, los sectores relacionados con alimentos, bebidas y limpieza aumentaron su demanda. Las familias necesitan adaptarse a una nueva realidad caracterizada por factores como el teletrabajo, hijos con clases online, preparar la comida diariamente, comprar productos esenciales por medio del comercio electrónico o por teléfono, realizar actividades recreativas en casa, celebraciones por videollamada, entre otros.

Este escenario de pandemia no fue exclusivo de Brasil, al contrario, afectó los hábitos y la rutina de millones de personas alrededor de todo el mundo de una forma abrupta, obligándolas a confinarse en casa sin ningún tipo de planificación previa. En los diferentes países, las empresas fueron afectadas de diversas maneras en cada segmento. Nicola et al. (2020) indican que el impacto de la pandemia se sintió en todos los sectores de la economía mundial, destacándose el Reino Unido. Este estudio de revisión indica que el sector de los alimentos, incluso la cadena de distribución y venta, en la que el embalaje posee un papel fundamental, sufrió un fuerte impacto durante los primeros meses de la pandemia. Los autores describen los cambios que ocurrieron en los hábitos de consumo en función de las compras por pánico y la necesidad de almacenar alimentos. Estas dos acciones por parte de los consumidores fueron las responsables del cambio en los hábitos de compras en relación con las categorías de productos, el tamaño de los envases y los sistemas de entrega. Hubo un aumento de 1.000 millones de libras en el Reino Unido en este segmento, lo que ocasionó que las empresas tuvieran que revisar sus procesos para satisfacer a una demanda creciente de entregas por medio de sistemas como el de delivery y un incremento en el consumo de productos con un mayor período de validez. El mismo fenómeno fue descrito por Hobbs (2020) con respecto a la cadena de alimentos de Canadá, que al igual que otras economías occidentales, está dominada por grandes cadenas de hipermercados con gran poder de compra, que basan sus decisiones en la eficiencia del costo y adoptan un sistema justo a tiempo. Según el autor, cuando ocurrió la crisis denominada compras de pánico, el sistema se mostró ineficiente para responder de forma rápida a un aumento del consumo, lo que generó un gran desabastecimiento 
y nerviosismo en la cadena alimentaria. Además de este hecho, destaca que el sistema de teletrabajo adoptado por varias empresas, y adoptado posteriormente en Brasil, provocó una disminución significativa en la alimentación fuera de casa, en lugares como restaurantes, bares, cafeterías, que son responsables de un tercio del consumo de alimentos en el país. También en Canadá, se registró un aumento de las ventas online de productos alimenticios y una tendencia de los consumidores a priorizar la compra de productos locales.

En Brasil, 37,5\% de las empresas reportaron que la pandemia tuvo un efecto negativo en los negocios, mientras que $26,1 \%$ informaron un efecto positivo; $33,1 \%$ tuvo dificultades para fabricar sus productos y atender a los clientes; $45,3 \%$ tuvo dificultades para ingresar proveedores de materias primas (Instituto Brasileño de Geografía y Estadística, 2020). La industria brasileña de alimentos y bebidas registró un aumento de o,8\% en la facturación y 2,7\% en la producción durante el primer semestre de 2020 comparado con 2019, impulsado por el aumento del consumo de las familias.

Sin embargo, el canal de food service percibió una caída de 29,5\% (Asociación Brasileña de la Industria de Alimentos - ABIA, 2020). Los productores locales de alimentos contribuyeron con el mantenimiento de la cadena de transporte vial, mientras que el transporte puerta a puerta de corta distancia realizado por medio de motos y bicicletas aumentó junto con las compras del canal e-commerce (Assunção, Medeiros, Moreira, Paiva \& Paes, 2020). Las empresas tuvieron que adoptar nuevas estrategias para vender productos $y$ servicios en el ámbito virtual, han utilizado diferentes estrategias como, por ejemplo, implementar y mejorar marketplaces, un sitio propio, redes sociales o la aplicación WhatsApp (Rezende, Marcelino \& Miyaji, 2020).

Aunque cada país tuvo una respuesta diferente a la pandemia en sus diferentes sectores, y muchas veces hubo diferencias significativas entre regiones dentro de un mismo país. Los pocos ejemplos internacionales antes mencionados indican que algunos puntos en común pueden verificarse en la tendencia de los nuevos hábitos de consumo de estos últimos cinco meses. Se considera importante entender el impacto local en función de las nuevas demandas producto de la emergencia, así como también de los cambios ocurridos en el comercio mundial. Las restricciones de importación y exportación de productos, dificultades logísticas para el recorrido de grandes distancias para la entrega en países de dimensiones continentales como Brasil, Canadá, Estados Unidos de América, entre otros, y la dependencia de la producción local de insumos importados, tanto para la cadena de producción de alimentos como para la cadena de envases, son los puntos más importantes para comprender el escenario de Brasil.

Aquí se entiende la cadena de envases, como la define la Associação Brasileira Embalagem - ABRE (2020), compuesta por fabricantes de materias primas para embalajes: acero, aluminio, plásticas, papel, cartón corrugado, vidrio y madera; fabricantes de tinta para la impresión de embalajes, adhesivos para el sellado, solventes de producción industrial, insumos y componentes fabriles; fabricantes de tapas y etiquetas; fabricantes de 
envases de diferentes materiales destinados al condicionamiento de los más diversos productos de uso esencial; distribuidoras logísticas.

El estudio de los hábitos del consumidor es una premisa en cualquier proyecto de embalaje, ya que el estudio dirigido a un determinado público forma parte de la etapa de recopilación de datos en el proceso de Diseño. Tiene como objetivo entender las necesidades y los deseos de los usuarios en relación con los envases (Merino, Merino \& Carvalho, 2009). Después de algunas semanas, luego del comienzo del aislamiento social, empresas del sector de envases y alimentos en Brasil, como también consultorías de tendencias, obtuvieron los primeros datos de consumo para analizar si hubo o no cambios relevantes en el perfil de consumo de los brasileños.

Este artículo presenta un compilado de noticias e investigaciones relacionadas con los cambios de hábitos de consumo de productos alimenticios en Brasil durante el período que se extiende de marzo a julio de 2020 , cuando el mundo enfrentó la pandemia de Covid-19, que fueron difundidos en las redes sociales especializadas, con el objetivo de presentar un escenario de este período que pueda brindar asistencia en el Diseño de envases.

\section{Metodología y desarrollo}

Se trata de un estudio exploratorio bibliográfico (Gray, 2012) que utilizó como fuentes de investigación noticias difundidas por portales de noticias, blogs, sitios y revistas online de asociaciones sectoriales, órganos gubernamentales e institutos de investigación especializados en el mercado de consumo, así como también la opinión de especialistas del sector de envases y alimentos difundidos en webinars en línea promovidos por la Feria de Insumos para Alimentos (FISPAL) en la plataforma Youtube. Para establecer un contexto comparativo del panorama brasileño con lo internacional también fue hecha una búsqueda en periódicos presentes en las bases de datos académicas Science Direct, BioResources, CityLibrary, GoogleScholar y Taylor of Francis. Las informaciones utilizadas para el análisis fueron publicadas entre el I de marzo y el 20 de junio de 2020 y estaban disponibles en formato de texto o video. Se realizó un análisis descriptivo de las informaciones obtenidas, de modo de establecer un escenario actualizado que involucra tres puntos de vista: las condiciones económicas locales, los cambios de comportamiento del consumidor brasileño en el período y las alteraciones percibidas y propuestas de las empresas y asociaciones del sector. Luego del análisis descriptivo fue elaborado un cuadro que describe la situación actual en Brasil relacionado con los cambios identificados en los hábitos de consumo y las posibles soluciones que pueden ser empleadas en nuevos diseños de envases de alimentos que satisfacen las necesidades de los consumidores en una situación de pandemia, aislamiento social y restricciones de movilidad.

\section{Diseño de envases}

Los principios teóricos del Diseño de envases que utilizamos actualmente se basan en presupuestos que emergieron después de la Segunda Guerra Mundial, en el período entre 1940-1959 denominado Self-Service Revolution por Opie (1989). Destacan la producción en masa, la comercialización en tiendas de autoservicio, los cambios en los hábitos de consumo por 
causa de la migración de la población del campo a los centros urbanos, la evolución de los materiales de los embalajes que aumentaron la conveniencia y el aumento de la vida útil de los productos. Además, la proliferación de las marcas de la competencia, que se disputan la preferencia del consumidor, demandó una postura diferente por parte de los diseñadores en relación con lo que ocurría antes. Se hizo conocida entre los diseñadores de envases en Brasil la frase de Seragini: "el envase es la vendedora silenciosa", en alusión al cambio en la forma de comercialización de los productos con el traspaso progresivo del comercio minorista a las tiendas de autoservicio, a partir de la década de 1950 (Negrão \& Camargo, 2008). Actualmente, sin embargo, vemos transformaciones provocadas por el uso cada vez mayor de tecnologías digitales por la sociedad mundial, y esto impacta de un modo significativo principalmente en la alteración del sistema de producción, venta y consumo de productos, agrega o altera los presupuestos del proyecto y la relación entre el producto, el consumidor y el envase (Mazurek \& Tkaczyk, 2016). La pandemia de Covid-19 aceleró estos cambios con el aumento exponencial de las ventas en los supermercados online, de modo que la decisión de compra en el punto de venta físico dejó de ser exclusiva.

Las etapas en el Diseño del envase se organizan en esta secuencia: búsqueda / investigación; definición de requisitos; creación de propuestas; finalización del proyecto.

Cuando se trata de un producto vendido en tiendas de autoservicio, existe una búsqueda intensa sobre el punto de venta físico con el objetivo de analizar los envases de los productos de la competencia, la disposición del producto en la góndola y el comportamiento del consumidor en el punto de venta (Bergmiller, 1976; Seragini, 1976; Mestriner, 2002; Merino, Merino \& Carvalho, 2009). Algunas empresas utilizan hasta técnicas de búsquedas neurológicas con el uso de la herramienta eye-tracking para identificar los puntos de más atención cuando el consumidor se encuentra delante de la góndola (Burger \& Knoll, 2018). Este énfasis en el punto de venta físico ha generado requisitos de proyecto que incluyen: destacar el envase en la góndola para diferenciarse de la competencia, crear el proyecto gráfico con la consideración de los envases que se pueden encontrar juntos, jerarquizar la información con el objetivo de presentar los atributos de venta cuando el consumidor está con un envase en las manos (Calver, 2009). La migración del punto de venta físico al virtual trae nuevos desafíos al Diseño del envase.

\section{Resultados}

Las principales tendencias de comportamiento identificadas durante el período de aislamiento en Brasil por la pandemia de Covid-19, según lo informado por los reportajes, institutos de investigación y webinars consultados, como Larghi (2020), Lavado (2020), Kantar (2020), Villela (2020), RG Nutri (2020) y Fispal (2020), fueron:

a. el aumento de las ventas online en las que se destacan bienes de consumo no durables, además del ingreso de nuevos consumidores a este canal;

b. el aumento de las ventas en supermercados en general para abastecer a los consumidores confinados en sus hogares; 
c. la reducción de los ingresos de la población y mayor sensibilidad de precios;

d. la necesidad de limpiar los productos antes de guardarlos;

e. el aumento de personas que cocinan en sus hogares;

f. la percepción de que la alimentación ha empeorado en casa; y

g. el uso de vitaminas.

Los dos primeros temas mencionados siguen las tendencias internacionales ya mencionadas. El agravamiento de la crisis económica en el país, con una tasa de desempleo del $11,4 \%$ entre el 24 de mayo y el 30 de mayo de 2020 , según el Instituto Brasileño de Geografía y Estadística (IBGE, 2020), explica la mayor sensibilidad a los precios al elegir los productos. Para complementar los datos, identificó que el 38,7\% de los hogares reciben una ayuda de emergencia del gobierno en mayo de 2020, y que 30,2 millones de personas que tienen trabajo vieron sus ingresos reducidos en ese período (IBGE, 2020).

Las investigaciones indican la supervivencia del virus en superficies, como por ejemplo la de Pressman et al. (2020) publicada por la revista Nutrition Today, en la que los autores destacan que, al analizar 22 estudios, pueden afirmar que el nuevo coronavirus sobrevive en superficies inanimadas como el metal, el vidrio o el plástico, hasta por nueve días. Los autores recomiendan la desinfección de superficies con hipoclorito de sodio (0,1\%) o una solución de 0,5\% de peróxido de hidrógeno; aun el uso de alcohol 71\% reduce significativamente la infección de las superficies por Covid-19. Esto llevó a que los consumidores adopten medidas de higienización para sus compras antes del almacenamiento, como forma de protección familiar contra la contaminación.

Con el cierre de los restaurantes, cafeterías, panaderías y bares, con un total de 13 millones de personas que no van a trabajar, ya sea porque trabajan desde sus casas o porque no pueden trabajar por causa del distanciamiento social, asociado a la reducción de ingresos, es un resultado previsible que más personas cocinen en sus casas.

Respecto de los últimos dos temas, la percepción de un empeoramiento de la alimentación en casa y el uso de vitaminas, van en direcciones opuestas. Al intentar mejorar el sistema inmunológico con el consumo de vitaminas, para evitar el contagio, algunos aspectos como el aumento de la ansiedad mencionado en la investigación Design for Emergency (2020), pueden explicar la compulsión alimentaria, generalmente asociada a este tipo de sentimiento, detallado a continuación.

\section{Cambios de hábitos de consumo}

Los datos utilizados se dividieron en dos períodos, el pre-aislamiento social, que comprende el período entre el 13 y el 16 de marzo, y el período de la suspensión de actividades no esenciales entre el 20 de marzo y el 16 de abril, donde ya estaba vigente el aislamiento social decretado por los Estados y Municipios. En el escenario previo al aislamiento social, según una encuesta a 500 personas mayores de 18 años en todo Brasil entre el 13 y el 16 de marzo, se descubrió que el $78 \%$ de las personas declararon que salieron de sus hogares solo para lo necesario y el 23\% declaró que trabaja en casa (Kantar, 2020c). 


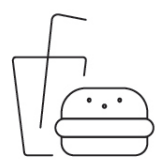

$17 \%$

Alimentos y Bebidas

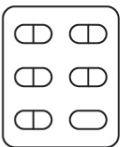

$15 \%$

Medicamentos sin prescripción médica

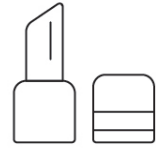

$12 \%$

Cosméticos e

Higiene Personal

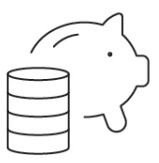

$12 \%$

Servicios

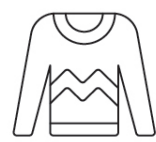

$\mathbf{8 \%}$

Ropa y Accesorios

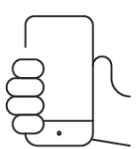

$8 \%$

Dispositivos

Electrónicos

El cambio más notable en este período fue el aumento del comercio online. En el sitio web de Mercado Libre, el número de nuevos compradores aumentó $28 \%$ entre el 24 de febrero y el 3 de mayo en comparación con el mismo período del año pasado (Larghi, 2020). Entre el 16 y el 23 de marzo, el aumento en las ventas de comercio electrónico fue del 100\% en relación con la semana anterior. La semana siguiente, del 24 al 31 de marzo, este aumento fue de $179 \%$ de acuerdo con una comparación semanal. Después, el aumento fue de $161 \%$ en la tercera semana entre el 1 y el 8 de abril, y alcanzó un aumento del $387 \%$ en la semana del 9 al 16 de abril en relación con la semana anterior (Larghi, 2020). En términos generales, el porcentaje de consumidores que compra online pasó de ser $19 \%$ a $34 \%$ entre los días 13 y 16 de marzo de 2020 (Kantar, 2020a).

Además del aumento exponencial del comercio electrónico, de acuerdo con André Dias, director de la empresa Compre\&Confie que monitorea ventas de más del $80 \%$ del comercio minorista digital brasileño, hubo un cambio en el perfil de compras (Villela, 2020). Normalmente, los brasileños estaban acostumbrados a comprar bienes duraderos con alto valor agregado, como teléfonos celulares, productos electrónicos, ropa y accesorios, pero del 24 de febrero al 25 de marzo, la venta de bienes no duraderos, como productos de salud, aumentó en $128,5 \%$ (Villela, 2020). Según la consultora E-bit, en la semana del 19 al 25 de marzo, el consumo minorista online de productos de consumo rápido casi se duplicó. Entre los ítems más buscados, los productos alimenticios básicos aumentaron $165 \%$, los productos fríos crecieron $106 \%$ y hortalizas, frutas y productos de granja 93\% (Lavado, 2020).

En el sitio web de Mercado Libre, entre el 17 y el 31 de marzo, la primera semana del período de cuarentena indicado por el Ministerio de Salud, la búsqueda de productos de higiene personal, alimentos y bebidas, farmacia y limpieza aumentó 64\% en comparación con 10 días antes (Lavado, 2020). Entre el 15 y el 24 de marzo, en comparación con los primeros diez días del mes, la venta de alimentos y bebidas creció un $40 \%$; en la categoría de equipos médicos y de salud, el aumento fue del $89 \%$; los artículos y muebles para el hogar aumentaron 93\%. Los artículos más comprados fueron los de cocina, decoración, aire acondicionado y muebles (Lavado, 2020). La Figura 1 muestra lo que las personas compraron por primera vez a través del comercio electrónico durante el período del 16 al 23 de marzo.

Figura 1. Compras realizadas por primera vez por categoría. Fuente: Kantar, $2020^{\mathrm{a}}$, p.2. 
Figura 2. Cambios en los hábitos de compra. Fuente: Kantar, 2020b, p.4.

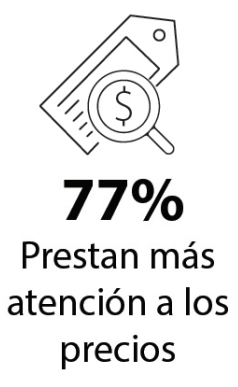

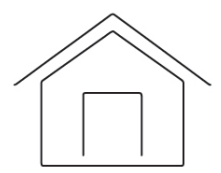

$75 \%$
Compran en mercados más pequeños cerca de sus casas

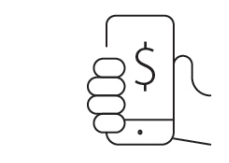

$68 \%$

Prefieren utilizar pago electrónico
En Brasil no hubo una política nacional de cuarentena o lockdown; la decisión de restringir el acceso a los servicios y el comercio, así como restricción de movilidad, quedó a cargo de los estados y municipios. Esto ocurrió por decisión judicial el 15 de abril (Falcão \& Vivas, 2020). Con el comercio minorista presencial en funcionamiento normal, hubo un cambio en el perfil de los consumidores, como se muestra en la Figura 2, donde el $77 \%$ de las personas son más sensibles al precio y analizan el mejor costobeneficio de los productos, el $75 \%$ prefiere comprar en supermercados cerca de casa para evitar las aglomeraciones, y el $68 \%$ prefiere los métodos de pago digital sobre el papel moneda (Kantar, 2020b).

Incluso con el crecimiento del comercio electrónico, en febrero las ventas de supermercados tuvieron un crecimiento real del 4,61\% en relación con enero, y el segmento de autoservicio comenzó el 2020 con uno de los resultados más altos de los últimos 9 años, un crecimiento de 5,11\% (Larghi, 2020). Esto ratifica otra investigación en la que los supermercados aún son en Brasil los principales lugares de compra de alimentos, responsables del $55 \%$ de las ventas durante la pandemia, seguidos por los mercados barriales con el $18 \%$ (RG Nutri, 2020).

Durante este período hubo cambios en el hábito de cocinar: aproximadamente 4 de cada 10 personas encuestadas comenzaron a cocinar o aumentaron su frecuencia en la cocina (RG Nutri, 2020). Esto se corrobora por el mayor acceso al contenido culinario en las redes sociales, que fue $45 \%$ más alto en el último mes en comparación con marzo de 2019, según los datos publicados por la plataforma YouPix (Borges, 2020). Para adaptarse a esta tendencia, la cadena de franquicias de comida italiana Spoleto comenzó a vender kits con los ingredientes para preparar los platos que se venden en los restaurantes para que los consumidores los prepararan en casa (Chuva, 2020). Aunque las personas cocinen en casa, existe la percepción de que la calidad de los alimentos ha empeorado: el $55 \%$ cree que comer en casa no es saludable y el $12 \%$ lo considera poco saludable en la cuarta semana de aislamiento social (RG Nutri, 2020) ([en el sentido de que consideran que comer en casa no es saludable] la gente come alimentos de baja calidad). Con respecto a la preocupación nutricional, $20 \%$ de los brasileños declararon usar suplementos y vitaminas: los complejos de vitamínicos y la vitamina $D$ crecen, y el uso de vitamina C disminuye (RG Nutri, 2020). 


\section{Nuevos hábitos de los consumidores e implicaciones en los envases}

Con respecto a la desinfección de los embalajes, la pandemia creó un nuevo hábito entre los consumidores que comenzaron a desinfectar todos los envases antes de guardarlos en los armarios o en el refrigerador. Se recomienda que los envases de alimentos industrializados se limpien con un paño empapado de alcohol gel o jabón. Los productos con envases como productos enlatados deben lavarse con jabón y agua corriente. Para las verduras, frutas y verduras, deben lavarse con agua corriente y colocarse sumergidas en una solución con cloro (Gontijo, 2020). De acuerdo con Ana Pontes de la empresa de alimentos Cargill, los envases que pueden ser higienizados con más facilidad deben ser priorizados por los consumidores, información corroborada por Adão Parra de la empresa de envases Diadema (Fispal, 2020). En este sentido, el envases de plástico flexible se vuelve más atractivo que el envases de papel. En general, existe una preferencia del consumidor por los envases sellados e inviolables, por lo que hubo una disminución en la venta de alimentos a granel según Cesar Freire de la empresa de bienes de consumo Unilever (Fispal, 2020).

En cuanto al tamaño de los envases, según Luciana Pellegrino, directora de la Associação Brasileira Embalagem - ABRE, hubo un aumento en el consumo de envases de tamaño familiar y una disminución en los envases para el autoservicio de alimentos y una sola porción. Esto puede explicarse por el cierre de bares y restaurantes, los principales clientes del segmento de autoservicio de alimentos y los paquetes más pequeños que se utilizan para el consumo fuera del hogar. Otro hecho que corrobora este fenómeno fue presentado por Kleber Mendonça de la empresa de alimentos Predilecta, quien señala que las personas cocinan más en casa y es por eso que prefieren envasar en porciones familiares y reutilizables (Fispal, 2020). Vivian Hang, de la empresa TetraPak, declaró que el envase de 1 litro aumentó las ventas en relación con los paquetes más pequeños (Fispal, 2020).

Uno de los impactos del e-commerce destacado por Ana Pontes de Cargill y Kleber Mendoça de Predilecta fue el de la distribución de los productos. Hubo un cambio en el flujo logístico normal, los productos son enviados por la empresa de alimentos a un centro de distribución y después son entregados a las tiendas para que, finalmente, el consumidor lo lleve de la tienda a su casa. Sin embargo, el comercio online sumó una nueva etapa en su logística, en la cual el pedido del cliente es colocado por el mercado online en una caja de embarque y entregado por una empresa tercerizada en la casa del consumidor. De esta manera, los paquetes de productos con diferentes pesos, formatos y categorías se agrupan dentro de la misma caja de envío y están sujetos a más pasos de manipulación e impactos de transporte imprevistos (Fispal, 2020). Para Kleber Mendonça, será un desafío para las empresas desarrollar un embalaje que llegue al consumidor intacto a través de este canal de ventas y mantener costos competitivos (Fispal, 2020).

Otro aspecto del e-commerce destacado por el diseñador Mario Narita es la comunicación del packaging. Se ve afectada la función del embalaje de atraer al consumidor y destacarse en el punto de venta. El punto de venta ahora virtual y el envase presentado en fotos reducidas, interfiere en la 
Tabla 1. Cambios de hábitos e influencia en los embalajes de alimentos.

Fuente: elaboración de los autores.
Envases más resistentes

Aumento del comercio electrónico

Foco en la comunicación en el momento del uso Conectar los envases con los medios digitales (omnichannel)

Higienización de los productos

Envases seguros e inviolables

Envases que permitan ser lavados

e higienizados fácilmente

Utilizar materiales que eliminen el Covid-19

Envases en tamaño familiar

Aumento de personas que cocinan en sus hogares

Envases prácticos y reutilizables

Foco en comunicar el modo de usar el producto

Reducción de poder adquisitivo

Envases con un mejor costo-beneficio Envases adecuados para cada perfil familiar

Aumento de la búsqueda de alimentos nutritivos

Envases que preservan los nutrientes Comunicar los beneficios a los consumidores

experiencia sensorial hasta el momento en que el producto se entrega, que ya no es al momento de la compra. En este entorno virtual, el diseñador debe considerar otros aspectos para comunicarse con el consumidor, como la experiencia de abrir el embalaje y el uso del producto, así como las estrategias de comunicación omnichannel (Fispal, 2020).

\section{Conclusiones}

La pandemia afectó la rutina de gran parte de la población mundial y no fue diferente en Brasil. Los cambios en los hábitos de consumo, desencadenados por el aislamiento social, introdujeron una nueva dinámica en el sector de envases para alimentos con el objetivo de satisfacer las nuevas necesidades de la cadena de alimentos. Estos cambios incluyen puntos importantes que impactan en todas las etapas del proceso productivo y de comercialización de los productos. La migración acelerada hacia el e-commerce, la necesidad de sanitización de los envases, el aumento del número de personas que cocinan en sus hogares y la disminución de la renta familiar son los cambios fundamentales que serán consideradas en la replanificación del packaging para el sector alimenticio.

Hoobs (2020) señala, como una de las conclusiones de su artículo, la necesidad de una mejor comprensión de las acciones que motivan comportamientos de pánico de consumo y la necesidad de almacenar alimentos para poder responder más eficientemente a toda la cadena de alimentos, con el propósito de disminuir el efecto de estos comportamientos. En este sentido, hay una convergencia con los datos encontrados en los estudios hechos en Brasil, que indican un escenario de cambio muy significativo de los hábitos de consumo en el país, con un claro impacto en las decisiones de los diseños de los envases.

En la Tabla 1, resume estas tendencias de comportamiento de consumo en la pandemia e indica algunos posibles desdoblamientos que se observarán en nuevos proyectos de packaging. De este modo, se puede deducir que el escenario de Brasil durante el período de aislamiento social desde el inicio de la crisis sanitaria mundial hasta el período de aislamiento social comprendido en este estudio para el segmento de envases de alimentos, 
que hay muchos desafíos por delante para satisfacer las nuevas necesidades de los consumidores y muy poco tiempo para implementarlos. Esperamos que este estudio pueda derivar en algunas de las soluciones e impulsar nuevas investigaciones.

La pandemia de Covid-19 hizo que surgieran nuevos desafíos y también intensificó los cambios que estaban en curso en el área del Diseño de envases en Brasil. A partir de los cambios identificados en el mercado brasileño, fueron elaboradas sugerencias para el Diseño de envases con consideración a los requisitos de comunicación, selección de materiales, transporte y reducción de costos.

Para una comunicación satisfactoria, la información del producto debe ser presentada de forma legible y actualizada, con una tabla nutricional, ingredientes, modo de conservación, vencimiento, lote de fabricación, modo de uso, datos del fabricante y del distribuidor, además de la información comercial adecuada en un entorno digital. Este objetivo puede alcanzarse con el reemplazo de las fotos del e-commerce por prototipos 3D que permiten que el usuario vea el envase desde todos los ángulos. En referencia a la selección de materiales para el envase, se recomienda evaluar el potencial del uso de materiales que se desarrollan para combatir el Covid-19, principalmente, los envases plásticos flexibles. En cuanto al costo, desarrollar propuestas gráficas más simples que tengan menor costo de impresión, para organizar los envases vendidos por e-commerce y entregados directamente al consumidor final, agrupados con envases diferentes en la misma caja, que se encuentran sujetos a impactos imprevisibles en el trayecto hasta la casa del consumidor.

En resumen, es natural que una tecnología que genera un cambio de paradigma sea utilizada, en principio, desde el punto de vista de la tecnología antigua, así como las primeras telenovelas brasileñas replicaban la lógica del teatro y de la radio, y posteriormente fueron adaptándose y exploraron las posibilidades de los nuevos medios. El sector alimenticio fue uno de los que sufrió un gran impacto con la migración de clientes hacia el e-commerce, y generó un impacto real para el Diseño durante la pandemia de Covid-19. En este sentido, los diseños de los envases deben pensar en nuevos requisitos de proyectos para el medio digital. 


\section{Referencias}

Associação Brasileira da Indústria de Alimentos - ABIA. Releases https://www.abia.org.br/releases/industriade-alimentos-cresce-08-em-faturamento-noprimeiro-semestre-2020>. Acesso em: 11 set. 2020.

Associação Brasileira de Embalagem - ABRE. (2020, junio 26). Comunicado oficial da ABRE. https://www.abre.org. br/comunicado-oficial-da-abre/

Bergmiller, K. H. (1976). Manual para planejamento de embalagens. MINISTÉRIO DA INDÚSTRIA E DO COMÉRCIO.

Borges, L. (2020). A quarentena e o movimento de cozinhar em casa. Consumidor Moderno. https:// www.consumidormoderno.com.br/2020/05/15/ quarentena-cozinhar/

Burger, C.A.C.\& Knoll, G. F. (2018). Eye tracking: possibilidades de uso da ferramenta de rastreamento ocular na publicidade. Fronteiras - Estudos Midiáticos, 20(3). https://doi.org/10.4013/fem.2018.203.07

Calver, G. (2009). O que é design de embalagem? BOOKMAN. Chuva, A. P. (2020, marzo 30). Com mais gente cozinhando em casa, aumenta procura por gás de cozinha em Campo Grande. Midiamax. https://www.midiamax. com.br/cotidiano/2020/aumento-de-pessoascozinhando-em-casa-faz-procura-por-gas-subir-emcampo-grande

Design for Emergency. (2020). Analysis (Brazil) - Design for Emergency. https://www.designforemergency.com/ analysis/br

Falcão, M. \& Vivas, F. (2020, Abril 15). Supremo decide que estados e municípios têm poder para definir regras sobre isolamento / Política / G1. G1. https://gı.globo.com/ politica/noticia/2020/04/15/maioria-do-supremovota-a-favor-de-que-estados-e-municipios-editemnormas-sobre-isolamento.ghtml

Fispal. [Food Connection]. (2020, May 29). Novas embalagens para um NOVO consumo. https://www. youtube.com/watch?v=V39mWKeEivs

Gontijo, J. (2020, March 22). Manipular e higienizar corretamente os alimentos é essencial para conter o coronavírus. Estado de Minas. https://www.em.com. br/app/noticia/bem-viver/2020/03/22/interna_bem_ viver,1130896/manipular-e-higienizar-corretamenteos-alimentos-e-essencial-para-cont.shtml

Gray, D. E. (2012). Pesquisa no mundo real (2a ed.). PENSO.

Hobbs, J. E. (2020). Food supply chains during the COVID-19 pandemic. Canadian Journal of Agricultural Economics, 68, 171-176. https://doi.org/10.1111/cjag.12237

Instituto Brasileiro de Geografia e Estatística. (2020). PNADCOVID19 / IBGE. https://covid19.ibge.gov.br/pnad-covid/ Kantar. (2020a). Consumer Thermometer 6 -

Perspectivas do E-commerce e do Consumo de Mídia. https://br.kantar.com/mercado-e-política/saúde-eesporte/2020/thermometer-ed6/

Kantar. (2020b). Consumer Thermometer 4 - O poder da conveniência. https://br.kantar.com/mercado-epolítica/saúde-e-esporte/2020/thermometer-ed4/

Kantar. (2020c). Consumer Thermometer 2 - O impacto da quarentena nas marcas e no comportamento de consumo. https://br.kantar.com/mercado-e-política/ saúde-e-esporte/2020/thermometer-ed2/

Larghi, N. (2020). Compras on-line crescem na quarentena; veja produtos mais vendidos. Valor Investe. https://valorinveste.globo.com/objetivo/ gastar-bem/noticia/2020/05/15/compras-on-linecrescem-na-quarentena-veja-produtos-maisvendidos.ghtml

Lavado, T. (2020). Alimentos, farmácia, brinquedos: vendas on-line crescem durante quarentena e levam empresas a mudar rotinas. G1. https://g1.globo.com/ economia/tecnologia/noticia/2020/04/o6/alimentosfarmacia-brinquedos-vendas-on-line-crescemdurante-quarentena-e-levam-empresas-a-mudarrotinas.ghtml

Merino, E., Merino, G. \& Carvalho, L. R. (2009). Guia de Orientação para o Desenvolvimento de Embalagens. Revista D.: Design, Educação, Sociedade e Sustentabilidade, 2, 84-100.

Mazurek, G. \& Tkaczyk, J. (Eds.). (2016). The impact of the digital world on management and marketing. POLTEX.

Mestriner, F. (2002). Design de embalagem: curso básico. PRENTICE HALL.

Ministério da Saúde. (2020, June 23). Coronavírus Brasil. https://covid.saude.gov.br/

Negrão, C. \& Camargo, E.P. de. (2008). Design de embalagem, do marketing à produção. NOVATEC.

Nicola, M., Alsafi, Z., Sohrabi, C., Kerwan, A., Al-Jabir, A., Iosifidis, C., Agha, M. \& Agha, R. (2020). The socioeconomic implications of the coronavirus pandemic (Covid-19): A review. International Journal of Surgery, Vol. 78, 185-193. Elsevier Ltd. https://doi.org/10.1016/j. ijsu.2020.04.018

Opie, R. (1981). Packaging Source Book. A visual guide to a century of packaging design. MCDONALD ORBIS BOOK.

RG Nutri. (2020). Tracking Covid-19 Alimentação e bemestar. http://www.rgnutri.com.br

São Paulo. (2020). SP Contra o Novo Coronavírus / Governo do Estado de São Paulo. https://www.saopaulo.sp.gov. br/coronavirus/

Seragini, L. (1976). A embalagem - da criação ao consumidor. VI Semana Tecnológica de Artes Gráficas, 18, 2-17.

Villela, C. (2020, Abril 1). Coronavírus - Quarentena 
aumenta vendas por e-commerce. Jornal Do Comércio.

https://www.jornaldocomercio.com/_conteudo/

especiais/coronavirus/2020/03/731946-quarentena-

aumenta-vendas-por-e-commerce.html

World Health Organization. (2020). Coronavirus

disease (Covid-19) Highlights. https://www.

who.int/docs/default-source/coronaviruse/

situation-reports/20200607-covid-19-sitrep-139.

pdf?sfvrsn=79dc6do8_2 